\title{
Study of the $K_{L}^{0} \rightarrow \pi^{+} \pi^{-} \gamma$ Direct Emission Vertex
}

A. Alavi-Harati ${ }^{12}$, T. Alexopoulos ${ }^{12}$, M. Arenton ${ }^{11}$, K. Arisaka ${ }^{2}$, S. Averitte ${ }^{10}$, A.R. Barker ${ }^{5}$, L. Bellantoni ${ }^{7}$, A. Bellavance ${ }^{9}$, J. Belz ${ }^{10, \dagger}$, R. Ben-David ${ }^{7}$, D.R. Bergman ${ }^{10}$, E. Blucher ${ }^{4}$, G.J. Bock ${ }^{7}$, C. Bown ${ }^{4}$, S. Bright ${ }^{4}$, E. Cheu ${ }^{1}$, S. Childress ${ }^{7}$, R. Coleman ${ }^{7}$, M.D. Corcoran ${ }^{9}$, G. Corti $^{11}$, B. Cox $^{11}$, M.B. Crisler $^{7}$, A.R. Erwin ${ }^{12}$, R. Ford ${ }^{7}$, A. Glazov ${ }^{4}$, A. Golossanov ${ }^{11}$, G. Graham ${ }^{4}$, J. Graham ${ }^{4}$, K. $\operatorname{Hagan}^{11}$, E. Halkiadakis ${ }^{10}$, K. Hanagaki ${ }^{8}$, S. Hidaka ${ }^{8}$, Y.B. Hsiung ${ }^{7}$, V. Jejer ${ }^{11}$, D.A. Jensen ${ }^{7}$, R. Kessler ${ }^{4}$, H.G.E. Kobrak ${ }^{3}$, J. LaDue ${ }^{5}$, A. Lath ${ }^{10}$, A. Ledovskoy ${ }^{11}$, P.L. McBride ${ }^{7}$, P. Mikelsons ${ }^{5}$, E. Monnier ${ }^{4, *}$,

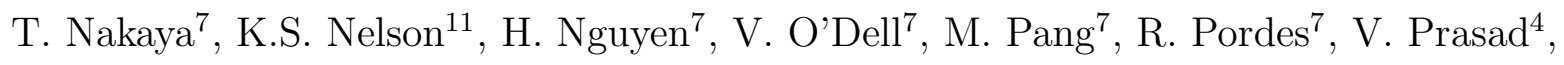
C. Qiao ${ }^{4}$, B. Quinn ${ }^{4}$, E.J. Ramberg ${ }^{7}$, R.E. Ray ${ }^{7}$, A. Roodman ${ }^{4}$, M. Sadamoto ${ }^{8}$, S. Schnetzer ${ }^{10}$, K. Senyo ${ }^{8}$, P. Shanahan ${ }^{7}$, P.S. Shawhan ${ }^{4}$, W. Slater ${ }^{2}$, N. Solomey ${ }^{4}$, S.V. Somalwar ${ }^{10}$, R.L. Stone ${ }^{10}$, I. Suzuki ${ }^{8}$, E.C. Swallow ${ }^{4,6}$, S.A. Taegar ${ }^{1}$, R.J. Tesarek ${ }^{10}$, G.B. Thomson ${ }^{10}$, P.A. Toale ${ }^{5}$, A. Tripathi ${ }^{2}$, R. Tschirhart ${ }^{7}$, Y.W. Wah ${ }^{4}$, J. Wang $^{1}$, H.B. White ${ }^{7}$, J. Whitmore ${ }^{7}$, B. Winstein ${ }^{4}$, R. Winston ${ }^{4}$, T. Yamanaka ${ }^{8}$, E.D. Zimmerman ${ }^{4}$

(KTeV Collaboration)

1 University of Arizona, Tucson, Arizona 85721

2 University of California at Los Angeles, Los Angeles, California 90095

3 University of California at San Diego, La Jolla, California 92093

4 The Enrico Fermi Institute, The University of Chicago, Chicago, Illinois 60637

5 University of Colorado, Boulder, Colorado 80309

${ }^{6}$ Elmhurst College, Elmhurst, Illinois 60126

7 Fermi National Accelerator Laboratory, Batavia, Illinois 60510

8 Osaka University, Toyonaka, Osaka 560 Japan

${ }^{9}$ Rice University, Houston, Texas 77005

10 Rutgers University, Piscataway, New Jersey 08855

11 The University of Virginia, Charlottesville, Virginia 22901 
12 University of Wisconsin, Madison, Wisconsin 53706

\begin{abstract}
We have perfomed studies of the $K_{L}^{0} \rightarrow \pi^{+} \pi^{-} \gamma$ Direct Emission (DE) and Inner Bremsstrahlung (IB) vertices, based on data collected by $\mathrm{KTeV}$ during the 1996 Fermilab fixed target run. We find $a_{1} / a_{2}=-0.737 \pm 0.034 \mathrm{GeV}^{2}$ for the $\mathrm{DE}$ form-factor parameter in the $\rho$-propagator parametrization, and report on fits of the form-factor to linear and quadratic functions as well. We concurrently measure $\Gamma\left(K_{L}^{0} \rightarrow \pi^{+} \pi^{-} \gamma, E_{\gamma}^{*}>20 \mathrm{MeV}\right) / \Gamma\left(K_{L}^{0} \rightarrow \pi^{+} \pi^{-}\right)=$ $(20.8 \pm 0.3) \times 10^{-3}$, and a $K_{L}^{0} \rightarrow \pi^{+} \pi^{-} \gamma \mathrm{DE} /(\mathrm{DE}+\mathrm{IB})$ branching ratio of $0.683 \pm 0.011$.
\end{abstract}

PACS numbers: 11.30.Er, 13.25.Es, 13.40.Hq, 14.40.Aq 
The decay $K_{L}^{0} \rightarrow \pi^{+} \pi^{-} \gamma$ (Figure 1) is a potential new window into the phenomenon of $\mathrm{CP}$-violation [1 [3]. This decay arises primarily from the $\mathrm{CP}$-violating Electric Dipole (E1) "Inner Bremsstrahlung" (IB) and the CP-conserving Magnetic Dipole (M1) "Direct Emission" (DE) processes. $\mathrm{KTeV}$ recently reported [if] the observation of a $\mathrm{CP}$-violating angular asymmetry between the $\pi^{+} \pi^{-}$and $e^{+} e^{-}$decay planes in the $K_{L}^{0} \rightarrow \pi^{+} \pi^{-} \gamma^{*}, \gamma^{*} \rightarrow$ $e^{+} e^{-}$mode, arising from the interference of $\mathrm{DE}$ and IB photon polarization states [5 [7]. If an E1 DE term were also present, this would generate a direct $\mathrm{CP}$-violating effect.

Understanding the precise photon energy $\left(E_{\gamma}^{*}\right)$ spectrum from the DE amplitude is crucial to interpretation of $\mathrm{CP}$-violating effects and may shed light on particular chiral models [8]. Previous experiments [9, 10] have observed evidence for an energy shift in the DE $E_{\gamma}^{*}$ spectrum, interpreted as evidence for an $E_{\gamma}^{*}$-dependent form-factor modification to the pure-M1 DE amplitude. Here we report on the first measurement of this form-factor from direct fits to the data, using the rare decay mode $K_{L}^{0} \rightarrow \pi^{+} \pi^{-} \gamma$.

We consider two separate form-factor parametrizations. Historically, the $\rho$-propagator form [8]

$$
\mathcal{F}=\frac{a_{1}}{\left(m_{\rho}^{2}-m_{K}^{2}\right)+2 m_{K} E_{\gamma}^{*}}+a_{2}
$$

has been used, where $a_{1} / a_{2}$ is the quantity of interest. More generally the form factor may be expressed as a Taylor series in $E_{\gamma}^{*}$

$$
\mathcal{F}=\left(1+\frac{r E_{\gamma}^{*}}{M_{K}}+\frac{s E_{\gamma}^{* 2}}{M_{K}^{2}}+\cdots\right)
$$

where the interesting quantitites are the slope $r$ and the quadratic parameter $s$. Recent theoretical work [11, 12] has attempted to calculate these parameters.

The data presented here was collected by $\mathrm{KTeV}$ operating in the E832 configuration [13] during the 1996 Fermilab fixed target run. A proton beam of intensity $\sim 3 \times 10^{12}$ protons per 19 second spill incident at an angle of $4.8 \mathrm{mr}$ on a $\mathrm{BeO}$ target was employed to produce two nearly parallel $K_{L}^{0}$ beams. In E832, one of these beams was incident on an active $K_{S}^{0}$ regenerator. Data collected in the regenerator beam was ignored for this analysis, except 
as a check of our understanding of the IB decay amplitude. The configuration of the KTeV E832 spectrometer consisted of a vacuum decay region, a magnetic spectrometer with four drift chambers, photon vetoes, a trigger scintillator bank, a CsI electromagnetic calorimeter, and a muon veto bank.

Signal $K_{L}^{0} \rightarrow \pi^{+} \pi^{-} \gamma$ and normalization $K_{L}^{0} \rightarrow \pi^{+} \pi^{-}$candidates were selected from the two-charged-track trigger. Offline, the sample was further purified by requiring the presence of two reconstructed tracks with a good vertex $-\chi^{2}$ within the fiducial aperture of the detector, and no significant activity in the photon veto counters. The tracks' kinematics were required to be inconsistent with $\Lambda^{0} \rightarrow p^{+} \pi^{-}$decays. The energy deposited by the charged pions in the calorimeter was required to be less than $0.85 \times$ the spectrometer momentum in order to eliminate backgrounds from $K_{e 3}$ decays. Candidate events were required to have a decay vertex between 110 and 156 meters downstream of the target and a total energy between 20 and $160 \mathrm{GeV}$.

$K_{L}^{0} \rightarrow \pi^{+} \pi^{-} \gamma$ candidates were subject to the additional criterion of requiring that the quantity

$$
P_{\pi^{0}}^{2} \equiv \frac{\left(M_{K}^{2}-M_{\pi^{0}}^{2}-M_{\pi \pi}^{2}\right)^{2}-4 M_{\pi^{0}}^{2} M_{\pi \pi}^{2}-4 M_{K}^{2}\left(P_{T}^{2}\right)_{\pi \pi}}{4\left(\left(P_{T}^{2}\right)_{\pi \pi}+M_{\pi \pi}^{2}\right)}
$$

be negative, i.e. by requiring that the $\pi^{0}$ momentum be imaginary under a $K_{L}^{0} \rightarrow \pi^{+} \pi^{-} \pi^{0}$ hypothesis. This cut virtually eliminates the $K_{L}^{0} \rightarrow \pi^{+} \pi^{-} \pi^{0}$ background to the $K_{L}^{0} \rightarrow \pi^{+} \pi^{-} \gamma$ event sample. In addition, at least one non-track-associated cluster in the calorimeter was required to possess $\geq 1.5 \mathrm{GeV}$ of energy, and to be at least $3 \mathrm{~cm}$ removed from the calorimeter edges. This "photon cluster" had to be at least $20 \mathrm{~cm}$ from the nearest track projection to reject background from $K_{L}^{0} \rightarrow \pi^{+} \pi^{-}$events accompanied by pion hadronic showers in the calorimeter. The photon was required to have an energy of at least $20 \mathrm{MeV}$ in the three-body center of momentum.

Figures 2 (a) and (b) show the vacuum beam $K_{L, S}^{0} \rightarrow \pi^{+} \pi^{-} \gamma$ data after final cuts. A very clean signal of $8,669 K_{L, S}^{0} \rightarrow \pi^{+} \pi^{-} \gamma$ events is achieved, with a background of about 0.5\%. Approximately $0.6 \%$ of the events in the peak in Figure 2 are residual $K_{S}^{0} \rightarrow \pi^{+} \pi^{-} \gamma$ 
decays from $K_{S}^{0}$ 's generated at the target. A total of 4,482,706 $K_{L}^{0} \rightarrow \pi^{+} \pi^{-}$events were accumulated for the normalization sample with a $0.1 \%$ background.

To extract the Direct Emission form-factor, we consider the distribution in $E_{\gamma}^{*}$, the photon energy in the center of momentum (Figure 3). We wish to extract the relative contributions of the Inner Bremsstrahlung and DE terms, as well as the energy shift of the DE spectrum due to the presence of a form factor. We assume that E1 IB and form-factormodified M1 DE are the only significant contributions to the decay. The possibility of an interference term arising from the presence of an E1 DE contribution is also briefly discussed below.

We perform a MINUIT [14] $\chi^{2}$-minimization fit to combine Monte Carlo DE and IB $E_{\gamma}^{*}$ distributions, and extract the relative DE and IB contributions to the data as well as the DE form-factor. In the fit, the form factor parameters $\left(a_{1} / a_{2}\right.$ or $r$ and $\left.s\right)$ and the ratio

$$
f=\frac{\Gamma_{D E}}{\left(\Gamma_{D E}+\Gamma_{I B}\right)}
$$

are allowed to float simultaneously. $\mathrm{A} \chi^{2}$ is formed from comparison of the resultant $D E+I B$ summed Monte Carlo histogram with the data, and minimized to obtain the best fit result.

Table [I summarizes the numerical fitting results for the three form-factor parametrizations. For the Taylor series parametrization, fits were performed with the quadratic parameter both fixed at zero (floating a single parameter, $r_{1}$ ) and allowed to vary (floating two parameters, $r_{2}$ and $s_{2}$ ). Data-Monte Carlo agreement in the $E_{\gamma}^{*}$ distribution is shown in Figure 3, for the $\rho$-propagator form. Figure 1 illustrates the expected effect of the various parametrizations on the pure M1 direct emission spectrum.

We see in the data presented in Table 1 and Figure 4 clear evidence for a modification to the pure-M1 DE spectrum. All fits are good, though the $\rho$-propagator hypothesis stands out slightly: It gives the best $\chi^{2}$ for a single-parameter fit, and the two-parameter fit results are in good agreement with the values $r_{2}=-2.70$ and $s_{2}=3.87$ obtained by Taylorexpanding the $\rho$-propagator form. The size of this energy shift, in particular the need to take into account terms of second-order in $E_{\gamma}^{*}$, is not currently understood within the chiral 
perturbation theory model for this decay [11].

Additional contributions to the photon energy spectrum are expected from $\mathrm{CP}$-violating higher-order multipole contributions to the Direct Emission amplitude. One possible consequence of these multipole terms is the presence of a charge-asymmetry in the $\pi^{+}$versus $\pi^{-}$Dalitz plot [1]. We exclude asymmetries larger than $2.4 \%$ at $90 \%$ c.l. with the present data.

One might also expect a contribution to the $E_{\gamma}^{*}$ spectrum from $\mathrm{CP}$-violating E1 DE, which could interfere with the E1 IB. We have allowed for such a term, by performing a separate fit in which we assumed an E1 DE amplitude constant in $E_{\gamma}^{*}$, and searched for the corresponding interference contribution [12] to the $E_{\gamma}^{*}$ spectrum. The form-factor parameter and $f$ are allowed to float simultaneously, while fixing the $I B$ rate at its theoretical value of $7.00 \times 10^{-3}$ 迎. Based on this fit, we set an upper limit of $\Gamma_{I N} /\left(\Gamma_{D E}+\Gamma_{I B}+\Gamma_{I N}\right) \leq$ 0.30 (90\% c.l.) on the contribution to the decay rate of the $\mathrm{CP}$-violating interference term. $\Gamma_{I N} / \Gamma_{A L L}=0.30$ corresponds to a $22 \%$ decrease in the $\Gamma_{D E} / \Gamma_{\mathrm{ALL}}$ ratio.

Background subtraction under the $E_{\gamma}^{*}$ distribution (with shape determined by study of $P_{T}^{2}$ sideband) resulted in no statistically significant change in either the form-factor parameter or the $D E /(D E+I B)$ ratio. Accidental activity in the detector was also found to have no statistically significant effect. The form factor parameters are found to have a slight $(1.9 \%)$ sensitivity to variations of the lower $E_{\gamma}^{*}$ cutoff (nominally $20 \mathrm{MeV}$ ) used in the fit. We also assign systematic uncertainties of $1.8 \%$ due to detector acceptance, and $1.4 \%$ due to the effects of uncertainties in the calorimeter photon energy scale. Bias due to Level 3 trigger inefficiency in the 1996 charged data set [13,15] is not significant in this analysis at the present level of statistics.

Note (Table $\mathbb{\mathbb { l }}$ ) that the measured $D E /(D E+I B)$ ratio is insensitive to the particular choice of form-factor parametrization. Uncertainty in the detector acceptance contributes a $0.7 \%$ systematic uncertainty to this ratio, while variations in analysis cuts contribute $0.6 \%$. The systematic uncertainty due to the calorimeter photon energy scale is $0.2 \%$.

We find the absolute $K_{L}^{0} \rightarrow \pi^{+} \pi^{-} \gamma$ branching ratio by normalizing the signal to the 
$K_{L}^{0} \rightarrow \pi^{+} \pi^{-}$channel. We determine the ratio of $K_{L}^{0} \rightarrow \pi^{+} \pi^{-} / K_{L}^{0} \rightarrow \pi^{+} \pi^{-} \gamma$ acceptances using a Monte Carlo simulation of the full detector and offline analysis criteria. Based on this, we calculate

$$
\frac{\Gamma\left(K_{L}^{0} \rightarrow \pi^{+} \pi^{-} \gamma\right)}{\Gamma\left(K_{L}^{0} \rightarrow \pi^{+} \pi^{-}\right)}=(20.8 \pm 0.2 \pm 0.2) \times 10^{-3}
$$

(where the first error is statistical and the second systematic) for $K_{L}^{0} \rightarrow \pi^{+} \pi^{-} \gamma$ events with $E_{\gamma}^{*}>20 \mathrm{MeV}$. The systematic uncertainty is due primarily to the effects of $K_{S}^{0}$ contamination in the vacuum beam. Assuming no contribution from interference and using

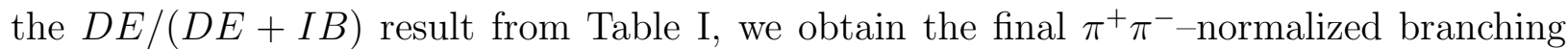
ratios $(14.2 \pm 0.2 \pm 0.2) \times 10^{-3}$ for Direct Emission and $(6.6 \pm 0.2 \pm 0.2) \times 10^{-3}$ for Inner Bremsstrahlung.

Our measured IB branching ratio is consistent with both the Q.E.D. prediction of $7.00 \times$ $10^{-3}$ and the most recent experimental result of FNAL E731 [10]; $(7.3 \pm 0.4) \times 10^{-3}$, based on a sample of $3,136 K_{L}^{0} \rightarrow \pi^{+} \pi^{-} \gamma$ events. The $D E /(D E+I B)$ ratio is also in good agreement with the E731 result of $0.685 \pm 0.041$, although the DE branching ratio differs from the E731 result of $(15.7 \pm 0.7) \times 10^{-3}$ by 1.8 standard deviations. The present form-factor results differ significantly from that reported by E731 $\left(a_{1} / a_{2}=-1.8 \pm 0.2 \mathrm{GeV}^{2}\right)$ but this discrepancy has been understood: The E731 $a_{1} / a_{2}$ form-factor was inferred [16] from the model of Lin and Valencia [8], on the basis of the measured $D E$ branching ratio, whereas our results are obtained directly by performing fits to the $E_{\gamma}^{*}$ distribution. The underlying datasets in the two experiments are consistent with each other, and a reanalysis of the E731 data using our method yields results consistent with ours. The $\mathrm{KTeV} K_{L}^{0} \rightarrow \pi^{+} \pi^{-} \gamma$ DE form-factor result is also in good agreement with the result $a_{1} / a_{2}=-0.720 \pm 0.029 \mathrm{GeV}^{2}$ extracted from the independent $K_{L}^{0} \rightarrow \pi^{+} \pi^{-} e^{+} e^{-}$analysis from the KTeV E799 data set [4].

In conclusion, we have made the first direct measurements of the $K_{L}^{0} \rightarrow \pi^{+} \pi^{-} \gamma$ Direct Emission form-factor, including $a_{1} / a_{2}=-0.737 \pm 0.034 \mathrm{GeV}^{2}$. We find no evidence for new $\mathrm{CP}$-violating effects in the photon energy spectrum. Finally, we have made improved measurements of the $\operatorname{DE}\left((14.2 \pm 0.2 \pm 0.2) \times 10^{-3}\right)$ and $\operatorname{IB}\left((6.6 \pm 0.2 \pm 0.2) \times 10^{-3}\right)$ 
$K_{L}^{0} \rightarrow \pi^{+} \pi^{-} \gamma$ branching ratios, normalized to the $K_{L}^{0} \rightarrow \pi^{+} \pi^{-}$channel.

We thank German Valencia and Jusak Tandean for discussions concerning this work. We gratefully acknowledge the support of the technical staff of Fermilab and participating institutions. This work was supported in part by the U.S. DOE, NSF, and The Ministry of Education and Science of Japan. 


\section{REFERENCES}

$\dagger \quad$ To whom correspondence should be addressed. Electronic address: belz@physics.montana.edu

* $\quad$ On leave from C.P.P. Marseille/C.N.R.S., France.

[1] G. Costa and P. K. Kabir, Nuovo Cimento 51A, 564 (1967).

[2] L. Sehgal and L. Wolfenstein, Phys. Rev. 162, 1362 (1967).

[3] G. D'Ambrosio and G. Isidori, Int. J. Mod. Phys. A13, 1 (1998).

[4] A. Alavi-Harati et al., Phys. Rev. Lett. 84, 408 (2000).

[5] L. Sehgal and M. Wanniger, Phys. Rev. D 46, 1035 (1992); Phys. Rev. D 46, 5209(E) (1992).

[6] P. Heiliger and L.M. Sehgal, Phys. Rev. D 48, 4146 (1993).

[7] J. K. Elwood et al., Phys. Rev. D52, 5095 (1995); J. K. Elwood et al., Phys. Rev. D53, 2855(E) (1996); J. K. Elwood et al., ibid., 4078 (1996).

[8] Y. C. R. Lin and G. Valencia, Phys. Rev. D37, 143 (1988).

[9] A. Carroll et al., Phys. Rev. Lett 44, 529 (1980).

[10] E. J. Ramberg et al., Phys. Rev. Lett. 70, 2525 (1993).

[11] G. Ecker, H. Neufeld, and A. Pich, Nucl. Phys. B413, 321 (1994).

[12] L. Littenberg and G. Valencia, Ann. Rev. Nucl. Part. Sci. 43, 729 (1993).

[13] A. Alavi-Harati et al., Phys. Rev. Lett. 83, 22 (1999).

[14] F. James, M. Roos, Comput. Phys. Commun. 10, 343 (1975).

[15] P. Shawhan, Ph.D. Thesis, University of Chicago 1999.

[16] E. Ramberg, private communication. 


\section{FIGURES}
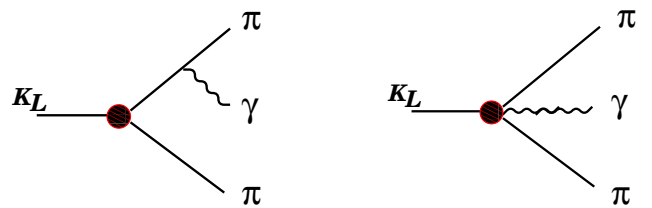

FIG. 1. The decay $K_{L}^{0} \rightarrow \pi^{+} \pi^{-} \gamma$ arises primarily from the contributions of the Electric Dipole (E1) "Inner Bremsstrahlung" (left) and Magnetic Dipole (M1) "Direct Emission" (right) diagrams above.
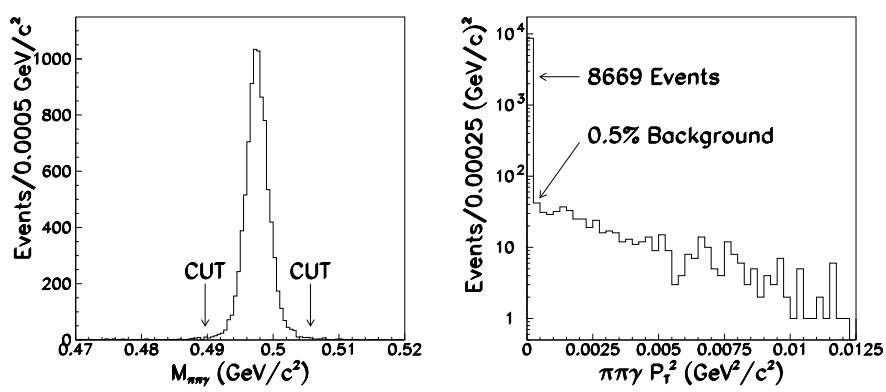

FIG. 2. a) $M_{\pi \pi \gamma}$ distribution of candidate $K_{L}^{0} \rightarrow \pi^{+} \pi^{-} \gamma$ events, all other cuts applied. Arrows indicate final cuts at $M_{k} \pm 8 \mathrm{MeV} / \mathrm{c}^{2}$. b) Kaon transverse momentum squared $\left(P_{T}^{2}\right)$ distribution of candidate $K_{L}^{0} \rightarrow \pi^{+} \pi^{-} \gamma$ events, all other cuts applied. The cut on this quantity requires events to be within the first bin $\left(0.000250 \mathrm{GeV}^{2} / \mathrm{c}^{2}\right)$ on this plot.

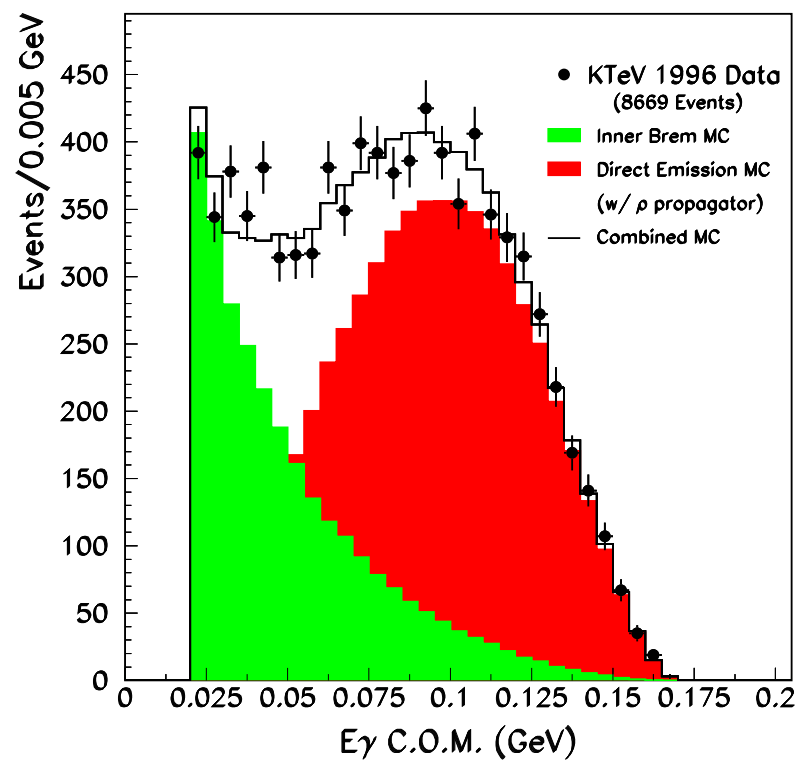


FIG. 3. $\quad K_{L}^{0} \rightarrow \pi^{+} \pi^{-} \gamma$ Monte Carlo/data overlay of photon energy distribution in the center of mass, for the best fit result. Shown also (shaded) are the expected distributions for pure E1 Inner-Bremsstrahlung and form-factor-modified M1 Direct Emission. The "combined" Monte Carlo plot shown assumes these two are the only contributions to the decay.

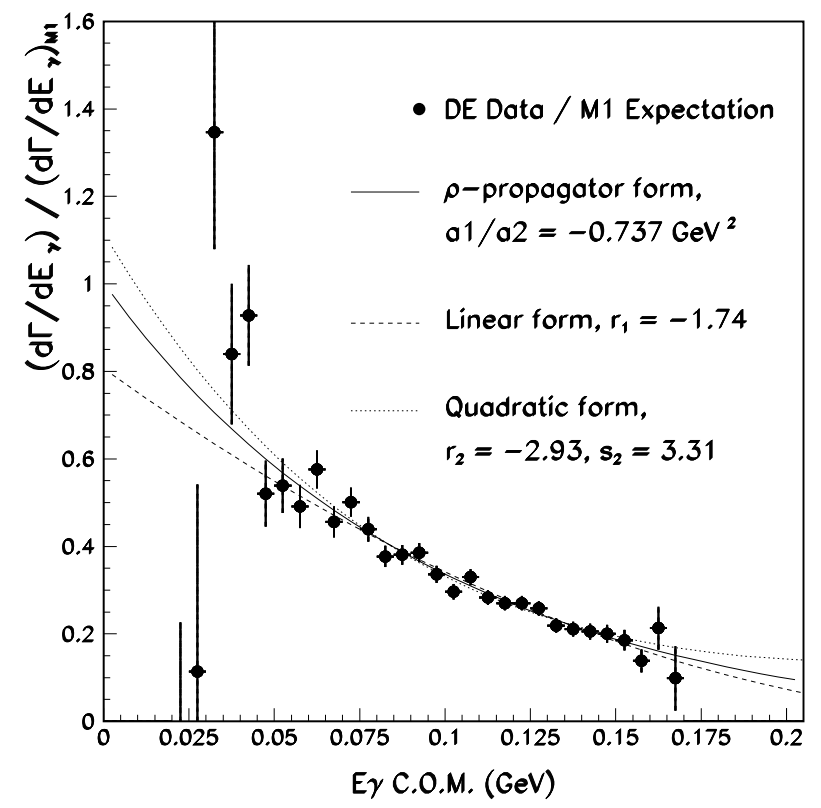

FIG. 4. Ratio (points) of (IB-Subtracted) Direct-Emission data to the expectation for a pure M1 $E_{\gamma}^{*}$ spectrum. Vertical scale is arbitrary. Shown for comparison are the $\rho$-propagator (solid), linear (dashed), and quadratic (dotted) form-factor parametrizations. A modification to the pure-M1 spectrum is clearly supported by the data. 


\section{TABLES}

\begin{tabular}{c|c|c|c|}
\hline \hline Quantity & $\rho$-propagator & Linear & Quadratic \\
\hline$\chi^{2} / \mathrm{DOF}$ & $38.8 / 27$ & $43.2 / 27$ & $37.6 / 26$ \\
$a_{1} / a_{2}$ & $(-0.737 \pm 0.026 \pm 0.022) \mathrm{GeV}^{2}$ & - & - \\
$r$ & - & $-1.739 \pm 0.062 \pm 0.052$ & $-2.93 \pm 0.41 \pm 0.34$ \\
$s$ & - & - & $3.31 \pm 1.15 \pm 0.96$ \\
$f$ & $0.683 \pm 0.009 \pm 0.007$ & $0.682 \pm 0.009 \pm 0.007$ & $0.684 \pm 0.011 \pm 0.007$ \\
\hline \hline
\end{tabular}

TABLE I. Summary of $K_{L}^{0} \rightarrow \pi^{+} \pi^{-} \gamma$ physics results for the three form-factor parametrizations. First uncertainty is statistical, second systematic. 